\title{
New Interpretations of The State Function of An Elementary Particle Based On The Origin of Quantum Probability
}

Shuming Wen ( $\nabla$ shmwen@126.com )

Kunming University of Science and Technology

\section{Research Article}

Keywords: Origin of probability, wave-particle duality, uncertainty principle, double-slit experiment, quantum tunnelling.

Posted Date: December 9th, 2021

DOI: https://doi.org/10.21203/rs.3.rs-1140095/v1

License: (c) (i) This work is licensed under a Creative Commons Attribution 4.0 International License.

Read Full License 


\title{
New Interpretations of the State Function of an Elementary Particle Based on the Origin of Quantum Probability
}

\author{
Shuming Wen
}

Faculty of Land Resources Engineering, Kunming University of Science and Technology, Kunming, China, 650093

\begin{abstract}
The theoretical results of quantum mechanics (QM) have been verified by experiments, but the probabilistic Copenhagen interpretation is still controversial, and many counterintuitive phenomena are still difficult to understand. To trace the origin of probability in QM, we construct the state function of a multiparticle quantum objective system and find that the probability in $\mathrm{QM}$ originates from the particle number distribution rate in a unit volume near position $r$ at time $t$ in the multiparticle quantum objective system. Based on the origin of probability, We find that the state function of the particle has precise physical meaning; that is, the particle periodically and alternately exhibits the particle state and wave state in time and space, obtain the localized and nonlocalized spatiotemporal range of the particle, the apparent trajectory of the particle motion. Based on this, through rigorous mathematical derivation and analysis, we propose new physical interpretations of the quantum superposition state, wave-particle duality, the double-slit experiment, the Heisenberg uncertainty principle, and the quantum tunnelling effect, and these interpretations are physically logical and not counterintuitive.
\end{abstract}

Kywords: Origin of probability, wave-particle duality, uncertainty principle, double-slit experiment, quantum tunnelling.

\section{Introduction}

As one of the pillars of modern physics, great achievements have been made with quantum mechanics (QM); the corresponding theoretical results are highly consistent with experimental results, and QM is the most accurate theory for describing microscopic particles. However, some problems about the physical nature of QM have been debated for decades and are still debated today. Some counterintuitive phenomena in QM, such as the quantum superposition state, wave function collapse, quantum tunnelling effect, uncertainty principle, and double-slit experimental phenomenon, have not been uniformly explained. For practical purposes, as long as the calculations are correct, there is no need to argue about the underlying physical mechanism. However, from the perspective of understanding nature, revealing the nature of the material world is a fundamental task of physics. Therefore, it is necessary to conduct further research on the physical interpretation of QM and in-depth exploration of the nature of the material world.

Regarding wave-particle duality, the quantum superposition state, wave function collapse, the quantum tunnelling effect, the uncertainty principle, and double-slit experiments, the physical Copenhagen interpretation [1] always results in some counterintuitive phenomena that defy physical logic, and these counterintuitive interpretations seem to be related to the probability in QM. Physicists such as Planck, Einstein, and Schrödinger were not satisfied with this probabilistic interpretation, leading to the so-called century of controversy over the physical interpretation of $\mathrm{QM}$, but they did not propose a more convincing interpretation. Although there are various counterintuitive results in the probabilistic Copenhagen interpretation, in recent decades, this interpretation seems to have become the orthodox interpretation of QM, and few people are as persistently opposed to it as Einstein and other 
scientists. Nevertheless, since 2016, Steven Weinberg[2], like Einstein and other physicists, has repeatedly raised questions. The integration of probability into physics has bothered physicists. However, the real difficulty of QM is not probability but the origin of probability. Therefore, to gain an in-depth understanding of these counterintuitive QM phenomena, it is particularly important to trace the origin of probability in QM. Based on the origin of the probability, we will obtain the new interpretations of the state function of an elementary particle.

\section{The origin of probability in QM}

\subsection{State function of a free particle}

Based on Einstein's photon hypothesis [3,4], de Broglie proposed that particles could be described not only as particles but also as waves [5]; i.e., for a free particle $\mathrm{j}$ with the energy of $E_{j}$ and momentum of $p_{j}$, its frequency and wavelength can be described as Equations (1) and (2), respectively, and its state can be described by a plane wave using a state function (Equation (3)).

$v_{j}=E_{j} / h$

$\lambda_{\mathrm{j}}=h / p_{j}$

$\psi_{j}=e^{i\left(p_{j} \cdot r-E_{j} t\right) / \hbar}$

where $v_{j}$ and $\lambda_{\mathrm{j}}$ are the frequency and wavelength of particle $\mathrm{j}$, respectively, $h$ and $\hbar$ are the Planck constant and the reduced Planck constant, respectively, $r$ and $t$ are the spatial position and time, respectively, and $\psi_{j}$ is the state function or wave function of particle $\mathrm{j}$.

\subsection{State function of a $\mathrm{N}$-free particle system}

A system composed of $\mathrm{N}$ free particles with a mass of $\mathrm{m}$ is called a multiparticle quantum objective system, such as multiple free-moving electronic systems after scattering by a crystal, multiple free-moving electronic systems fabricated at a certain voltage for double-slit experiments, and white light composed of multiple photons with different frequencies. The allowable states of the particle are labelled $1,2,3, \ldots \mathrm{j} \ldots$, the momentum of the particle in these states is labelled $p_{1,} p_{2}, p_{3} \ldots p_{j} \ldots$, the energy is labelled $E_{1}, E_{2}, E_{3} \ldots E_{j} \ldots$, the number of particles in these states is represented by $N_{1}, N_{2}, N_{3} \ldots N_{j} \ldots$, and the number of particles in some states is allowed to be 0 . Obviously, $N_{1}+N_{2}+N_{3}+\ldots+N_{j}+\ldots=N$. The proportion of the number of particles in state $\mathrm{j}$ to the total number of particles in the unit volume near position $r$ at time $t$ is $\left|C_{p_{j}}^{N}(r, t)\right|^{2}$, which is called the particle number distribution rate. In the whole space, $\iiint_{-\infty}^{+\infty}\left|C_{p_{j}}^{N}(r, t)\right|^{2} d x d y d z=N_{j} / N$. Based on the basic principle of linear superposition in waves, the state of a multiparticle quantum objective system can be described by the linear superposition state of all particle states; that is, the state function of the system can be expressed as Equation (4).

$\psi=\sum C_{p_{j}}^{N}(r, t) e^{i\left(p_{j} \cdot r-E_{j} t\right) / \hbar}, \mathrm{j}=1,2,3 \ldots(4)$

where $\psi$ represents the state function of the multiparticle quantum objective system and the squared modulus of the state function $|\psi|^{2}$ equals the particle number distribution rate in the unit volume near position $\mathrm{r}$ at time $\mathrm{t}$; that is, $|\psi|^{2}=\Sigma\left|C_{p_{j}}^{N}(r, t)\right|^{2}$.

Since the total number of particles in the entire space, N, remains unchanged, the particle 
number distribution rate in the entire space is always 1.

$\sum \iiint_{-\infty}^{+\infty}\left|C_{p_{j}}^{N}(r, t)\right|^{2} d x d y d z=\frac{N_{1}+N_{2}+N_{3}+\cdots+N_{j}+\cdots}{N}=1, \mathrm{j}=1,2,3 \ldots$ (5)

Obviously, for a quantum objective system with only one particle, based on Equation (4), the state function of this particle can also be expressed by Equation (6).

$\psi=\sum C_{p_{j}}^{1}(r, t) e^{i\left(p_{j} \cdot r-E_{j} t\right) / \hbar}, \mathrm{j}=1,2,3 \ldots(6)$

At the time-space point where this particle is located, the particle number distribution rate is 1 , and in other time-space regions, the particle number distribution rate is zero.

$\sum \iint_{-\infty}^{+\infty}\left|C_{p_{j}}^{1}(r, t)\right|^{2} d x d y d z=\frac{0+0+0+\cdots+1+\cdots}{1}=1, \mathrm{j}=1,2,3 \ldots(7)$

When the particle in the system is not a free particle, its state function is more complex than that of a free particle, but the state function of the system can still be transformed into the form of Equation (4) through Fourier transform.

\subsection{The origin of probability in QM}

Equation (4) is the state function of a multiparticle quantum objective system composed of all state particles with a mass of $\mathrm{m}$. Without considering the interaction between these particles, each particle has its own energy, momentum, and motion state. When considering a particle in this system, We cannot know which of the many particles in the system this particle is before the measurement since this system has many particles, so this particle can be any particle in the system. To facilitate the comprehensive study of this particle, we construct a QM system of one particle. Before the measurement, the particle in the QM system has the possibility of all allowed states in the multiparticle quantum objective system. If the particle has the state described by $\psi_{j}$, then the possibility of this particle having the state $\psi_{j}$ is $\iiint_{-\infty}^{+\infty}\left|C_{p_{j}}^{N}(r, t)\right|^{2} d x d y d z=N_{j} / N$, and the particle number distribution rate of the state $\psi_{j}$ in the entire space of the multiparticle quantum objective system is $\iiint_{-\infty}^{+\infty}\left|C_{p_{j}}^{N}(r, t)\right|^{2} d x d y d z=$ $N_{j} / N$, which is also the probability of this particle in the state $\psi_{j}$ in the QM system.

Thus, for a particle in a QM system, we can also use Equation (4) to represent its state function. To be distinguished from the state function of the multiparticle quantum objective system, the state function of this particle in the QM system is expressed as Equation (8).

$\psi=\sum C_{p_{j}}(r, t) e^{i\left(p_{j} \cdot r-E_{j} t\right) / \hbar}, \mathrm{j}=1,2,3 \ldots(8)$

where $\psi$ represents the state function of a particle in the QM system and the squared modulus of the state function $|\psi|^{2}$ is the particle number distribution rate in a unit volume near position $r$ at time $t$ in a multiparticle quantum objective system. In QM, this is called the probability of finding a particle in a unit volume near position $r$ at time $t$, we think, which is the origin of the probability of Born's statistical interpretation [6].

If the state function of a particle in QM can be treated as a continuous function, then Equation (8) can be expressed in an integral form, as shown in Equation (9).

$\psi=\frac{1}{(2 \pi \hbar)^{3 / 2}} \iiint_{-\infty}^{+\infty} C_{p}(r, t) e^{i(\boldsymbol{p} \cdot \boldsymbol{r}-E t) / \hbar} \mathrm{d} p_{x} d p_{y} d p_{z}(9)$

This state function is identical with the wave function of matter deduced by de Broglie ${ }^{5}$. 
From the point of view of mathematics and physics, Equations (8) and (9) show that before the measurement, we do not know the state of the studied particle, and we do not know whether the state of the particle is changing; therefore, all possible states of the particle should be considered, so the state function of a particle in QM is always the linear superposition state of all possible states before measurement, which is the physical and mathematical essence of the superposition principle of state functions.

In a multiparticle quantum objective system, particles in different states can initially be at different spatial positions and have different momentums and energies, i.e., different motion velocities, motion directions, and starting positions. With the evolution of time, these particles move in their respective directions at their respective velocities, the particle population can continue to expand in space, and the spatial volume occupied by particles can continue to expand, which is the physical and mathematical nature of the continuous expansion of the state function of a particle in space over time in QM.

Since the particle in the entire space can always exist in an allowed state, the total probability of the particle in the entire space in various allowed states is 1 .

$\sum \iiint_{-\infty}^{+\infty}\left|C_{p_{j}}(r, t)\right|^{2} d x d y d z=1, \mathrm{j}=1,2,3 \ldots(10)$

$\iiint_{-\infty}^{+\infty}|\psi|^{2} d x d y d z=1$

The state function of a multiparticle quantum objective system with a mass of $m$ and the state function of a particle in QM are both solutions to Schrödinger's equation, given in Equation (12) [7], and both undergo time evolution according to this equation.

$i \hbar \frac{\partial \psi}{\partial t}=-\frac{\hbar^{2}}{2 m} \nabla^{2} \psi+U \psi$

Since the multiparticle quantum objective system does not have the concept of probability, there is no randomness. However, the QM system introduces the concept of probability through artificial mathematical operations and thus introduces randomness.

\section{New physical interpretation of the state function}

\subsection{Physical meaning of the state function}

\subsubsection{The physical meaning of the state function and its superposition state Copenhagen interpretation:}

According to the Copenhagen interpretation, the state function of particles is also called the wave function. The wave function $\psi$ is the description of the quantum state of the system and is the probabilistic amplitude of the particle appearing at position $r$ at time $t$, and the squared modulus of the wave function $|\psi|^{2}$ represents the probability of the particle appearing in the unit volume near position $r$ at time $t$. The matter wave is only the probability wave without any physical reality.

The wave function of a particle $\psi$ can be expanded into a mathematical superposition state (Equation (8) or (9)) using the Fourier series; i.e., if $\psi_{1}, \psi_{2}, \psi_{3} \ldots \psi_{j}, \ldots$ are the possible states of the particle, then $\psi=\sum C_{p j} \psi_{j}, \mathrm{j}=1,2,3 \ldots$ indicates that the superposition state is also a possible state of the particle. In the superposition state, a particle can be in different spatial positions at the same time with different probabilities, and a particle can have different states at the same time with different probabilities.

Based on the probabilistic interpretation of the wave function by the Copenhagen 
interpretation, for a free particle with known momentum and energy, Equation (3) is the wave function $\psi_{j}$, and the squared modulus of the wave function $\left|\psi_{j}\right|^{2}=1$; that is, the probability of finding the particle at any position in space is 1 , this is obviously inconsistent with the facts.

Based on the probabilistic interpretation of the wave function by the Copenhagen interpretation, when the hydrogen atom is at the ground state, the squared modulus of the wave function is not 1 at the ground state radius $r_{1}$ and is not 0 at $0<r<r_{1}$ or $r_{1}<r<r_{2}$, which indicates that it is possible to find the electron anywhere in the region of $0<r<r_{2}$. This result obviously contradicts the conclusion stated in the hydrogen atom theory separately derived by Bohr, Heisenberg, de Broglie, and Schrödinger that the ground state electron can be found only at the position $r=r_{1}$. It is also inconsistent with the fact reflected by the hydrogen atomic spectrum.

Therefore, the probabilistic interpretation of the wave function by the Copenhagen interpretation is imperfect.

\section{New physical interpretation:}

Based on quantum field theory, we believe that the state function of a particle $\psi$ is the mathematical expression of its wave-particle duality, indicating that the particle periodically and alternately exhibits the particle state and wave state in time and space. In the space-time points of $p r=n h, E t=n h(\mathrm{n}=1,2,3 \ldots)$, the particle is in a particle state, while in the space-time region of $(\mathrm{n}-1) h<p r<n h,(\mathrm{n}-1) h<E t<n h(\mathrm{n}=1,2,3 \ldots)$, the particle is in a wave state.

According to the origin of probability, the superposition state of a particle state function shows: (1) when the momentum and energy of the particle are constant, $\left|C_{p_{j}}\right|^{2}$ represents the probability of the particle with the state $\psi_{j}$ near position $\mathrm{r}$ at time $\mathrm{t}$; (2) when the momentum and energy of the particle change with time and space, the particle is in a changing state between the two states $\psi_{j}$ and $\psi_{k}$ in the superposition state, and the state function of the particle at this time is $\psi_{j k}=C_{p_{j}} \psi_{j}+C_{p_{k}} \psi_{k}$, where $k \neq j, \mathrm{j}=1,2,3 \ldots, \mathrm{k}=1,2,3 \ldots$, with $\left|C_{p_{j}}\right|^{2}+\left|C_{p_{k}}\right|^{2}=1$. We also call the superposition state corresponding to $\psi_{j k}$ transition state.

\subsubsection{The physical nature of the superposition state collapse Copenhagen interpretation:}

The Copenhagen interpretation indicates that before the measurement, the wave function of the particle $\psi$ is in the superposition state (Equation (8) or (9)). When the operator $\hat{F}$ corresponding to a mechanical quantity is used on the wave function $\psi$, i.e., when the mechanical quantity of the particle is measured, the wave function $\psi$ collapses to the eigenstate of the particle $\mathrm{F}_{\mathrm{j}} \psi_{j}$, and the eigenvalue $\mathrm{F}_{\mathrm{j}}$ and eigenfunction $\psi_{j}$ of this measurement are obtained.

The physical nature of the superposition state collapse of the wave function is still unclear. In particular, before the measurement, the particle is at different spatial positions with different probabilities; during measurement, the particle suddenly collapses from various positions in space to a certain position, which seems to allow faster-than-light-speed events to occur.

New physical interpretation: 
(1) If the momentum and energy of a particle are constant, based on the origin of probability, we believe that a particle in a superposition state, regardless of measurement, is actually in a certain state $\psi_{j}$ in the superposition state. The measurement is only to find the actual state of the particle $\psi_{j}$ from the superposition state composed of all possible states, and the probability of finding the particle in the state $\psi_{j}$ in a unit volume near position $\mathrm{r}$ at time $\mathrm{t}$ is $\left|C_{p_{j}}\right|^{2}$. Once the particle is found to be in its actual state, the superposition state ceases to exist; i.e., the wave function collapses. The measurement of the state is only a mathematical selection process and does not involve any events related to state transitions or particle movements in the quantum objective system.

In measurement, the eigenstate of the particle $\mathrm{F}_{\mathrm{j}} \psi_{j}$ is found in the superposition state of the wave function $\psi$, and the eigenfunction of this measurement $\psi_{j}$ is obtained. However, it is possible that the eigenvalue $F_{j}$ cannot be obtained at the same time. Because the result of applying the operator is Equation (13), and Equation (13) shows that only if $p_{j} r=n h$ and $E_{j} t=n h(\mathrm{n}=1,2,3 \ldots)$, the result of Equation (13) equals to $F_{j}$. In other words, only when the particle is in the particle state can the measurement obtain the mechanical quantity of the particle. In the time-space region of $(\mathrm{n}-1) h<p_{j} r<n h,(\mathrm{n}-1) h<E_{j} t<n h(\mathrm{n}=1,2$, $3 . .$.$) , the particle is in a wave state, and the measurement cannot obtain any mechanical$ quantity of the particle.

$\widehat{\mathrm{F}} \psi=\mathrm{F}_{\mathrm{j}} \psi_{j}=F_{j} e^{i\left(p_{j} \cdot r-E_{j} t\right) / \hbar}$

(2) When the momentum and energy of the particle change, we believe that the use of operator $\hat{F}$ corresponding to the mechanical quantity on the wave function $\psi$, i.e., the measurement of the mechanical quantity of the particle, cannot obtain the eigenstate, eigenfunction, and eigenvalue. Because the actual state of the particle is $\psi_{j k}=C_{p_{j}} \psi_{j}+$ $C_{p_{k}} \psi_{k}$, the state of the particle changes and cannot be determined during the measurement. Therefore, the measurement cannot make a choice for the changing state. Without the measurement result, the superposition state (the transition state) does not collapse due to the non destructive measurement.

\subsection{Physical interpretation of wave-particle duality \\ Copenhagen interpretation:}

The Copenhagen interpretation indicates that all particles may be partially described not only in particle terms but also in wave terms, which means that the classical concepts of particles and waves cannot fully describe physical behaviour on the quantum scale, and the particle becomes a contradictory unity of wave and particle [8]. Equations (1) and (2) of de Broglie are mathematical expressions of the wave-particle duality of particles, and Equation (12) of Schrödinger reflects the evolution of the wave-particle duality with time.

When people observe particles using particle terms, the particles exhibit particle characteristics, and wave characteristics cannot be observed. When people observe particles using wave terms, the particles exhibit wave characteristics, and particle characteristics cannot be observed. The state of the particle depends on the method of observation. When there is no observation, the particle is in the superposition state of the wave and particle.

This understanding of wave-particle duality by the Copenhagen interpretation implies the 
possibility that human observations determine objective reality.

\section{New physical interpretation:}

We believe that all particles periodically and alternately exhibit the particle nature and wave nature in time and space. In the time-space points of $p r=n h, E t=n h(\mathrm{n}=1,2,3 \ldots)$, the particle is in the particle state, and in the time-space region of $(n-1) h<p r<n h$, $(\mathrm{n}-$ 1) $h<E t<n h(\mathrm{n}=1,2,3 \ldots)$, the particle exhibits a wave state. The state function of the particle $\psi$ is the mathematical expression of the wave-particle duality of the particle, and Equation (12) of Schrödinger reflects the evolution of the wave-particle duality of the particle over time.

When people make observations, if a particle is at a time-space point corresponding to the particle state, the particle exhibits particle characteristics, and people cannot observe wave characteristics; if the particle is in a space-time region corresponding to the wave state, the particle exhibits wave characteristics, and people cannot observe particle characteristics.

We believe that particle characteristics and wave characteristics are the alternating basic properties of particles and that observation cannot change the objective reality of particles being in particle state or wave state in time and space.

\subsection{Double-slit experiment-geometric image of wave-particle duality}

In the early 19th century, Thomas Young [9]first introduced the double-slit interference experiment of light. For more than one hundred years, countless double-slit interference experiments have obtained the same pattern. The experimental principles are shown in Fig. 1(a). A white (or red) light is projected at the double slits A and B with a distance of d. After passing through the double slits, the photons continuously hit the receiving screen OP, whose distance from the double slits is L; the obtained interference image is shown in Fig. 1(b) (or (Fig. 1(c)). Similarly, a group of electrons prepared according to certain conditions are emitted towards double slits A and B with a spacing of d. After passing through the double slits, the electrons continuously hit the receiving screen OP, whose distance from the double slits is L; the obtained image is shown in Fig. 1(d) [10]. When the electrons arrive at the receiving screen one by one, in the initial stage with very few electrons, several irregular dots are displayed on the receiving screen. As the number of electrons increases, the gradually formed image is almost the same as the image formed when all the electrons reach the receiving screen at the same time, as shown in Fig. 1(e).
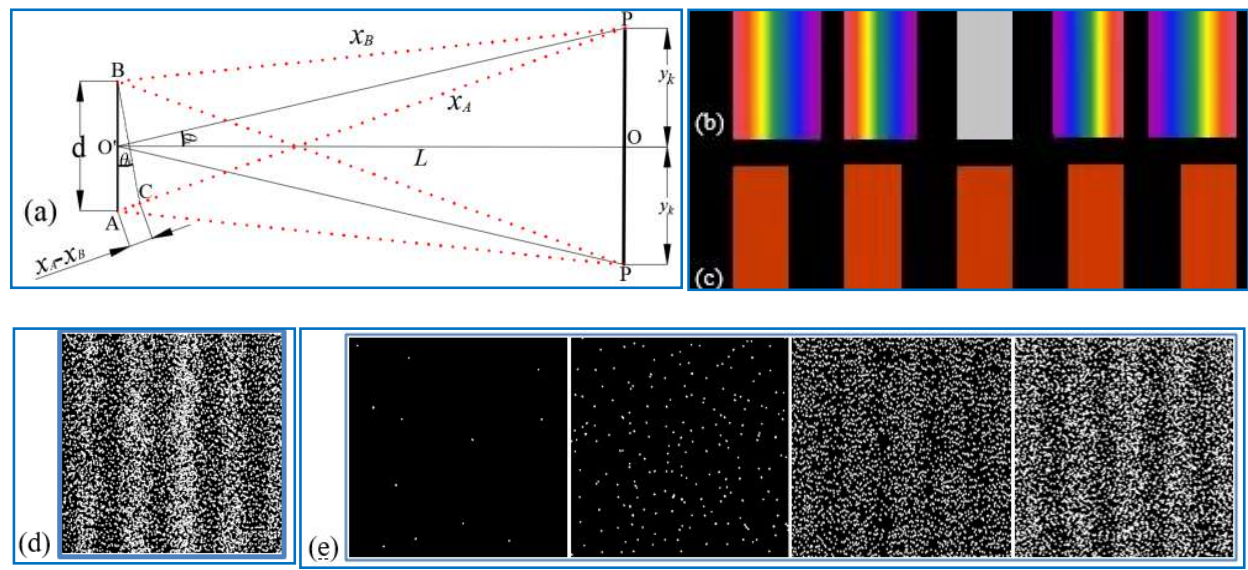

Fig. 1 Schematic diagram of the light or particle double-slit experiment

(a) Geometric relationships of light and particle double-slit experiment, (b) the simulation results of the 
white light double-slit experiment, (c) the simulation results of the red light double-slit experiment, (d) the simulation results of electrons arriving at the receiving screen together (e) the simulation results of electrons arriving at the receiving screen one by one

The results obtained by Young's double-slit interference experiment of white light in Fig. 1(b) have been satisfactorily explained by the wave nature of light; that is, the position where the bright fringe appears satisfies Equation (14) [9].

$d \sin \theta_{k}= \pm k \lambda, \mathrm{k}=0,1,2,3 \ldots(14)$

where $d$ is the double-slit spacing, $\theta_{k}$ is the angle of the bright fringe on the receiving screen from the centre of the receiving screen, and $\lambda$ is the wavelength of the light.

In QM, both light and particles have wave-particle duality, and the double-slit interference experiment of light is an experimental verification of the wave nature of light. The double-slit experiment of particles shows a phenomenon similar to that of the experiment of light, so the double-slit experiment of particles is used as an experimental verification of the wave nature of particles. However, there has been controversy about the probabilistic interpretation of the double-slit experiment by the Copenhagen interpretation.

\section{Copenhagen interpretation:}

The Copenhagen interpretation indicates that both photons and particles have wave-particle duality and that their wave function is $\psi$. Before a photon or particle is observed, its wave function is in a superposition state, so it is both a wave and a particle. In the double slit experiment, the photon or particle reaches a receiving screen through double slits, and the receiving screen is an instrument detecting the particle characteristics. The wave function of the photon or particle collapses to a point, and the receiving screen detects the photon or particle. The position of the photon or particle collapsing to a point on the receiving screen strictly follows the probability distribution of the superposition state of the wave function. The position of a photon or particle on the receiving screen is random, but the positions of multiple photons or particles on the receiving screen exhibit probability distribution-determined fringes.

This probabilistic Copenhagen interpretation is the orthodox interpretation of the double-slit experiment by QM. However, photons with different momenta have different wavelengths. Fig. 1(b) shows that the red photons, with long wavelengths, land away from the centre and that the blue photons, with short wavelengths, land closer to the centre. Photons of different wavelengths land at different positions in a bright fringe by strictly following the state distribution, and the wave function collapses into a bright fringe, forming a colour band with a certain width. However, in Fig. 1(b), there are multiple colour bands of equal distance. The photons of the same state (such as red photons) fall into different colour bands of equal distance, which cannot be explained by the probability distribution proposed by the Copenhagen interpretation, and the probabilistic Copenhagen interpretation also fails to obtain Equation (14), the fringe distribution of double-slit experiment.

Based on the principle of the state superposition of wave functions, the Copenhagen interpretation proposes that photons or particles passing through double slits $\mathrm{A}$ and $\mathrm{B}$ have two possible states of $\psi_{A}$ and $\psi_{B}$. When a photon or particle passes through double slits A and B, it passes through slits $\mathrm{A}$ and $\mathrm{B}$ at the same time in a superposition state shown in Equation (15). $\psi=\psi_{A}+\psi_{B}(15)$

When the photon or particle reaches the receiving screen, the wave function collapses, and the position of the photon or particle is determined by the squared modulus of the wave function, 
that is, the probability. Since $|\psi|^{2}=\left(\psi_{A}+\psi_{B}\right)^{2}=\left|\psi_{A}\right|^{2}+\left|\psi_{B}\right|^{2}+\psi_{A} \psi_{B}{ }^{*}+\psi_{B} \psi_{A}{ }^{*}$, the cross term $\psi_{B} \psi_{A}{ }^{*}+\psi_{A} \psi_{B}{ }^{*}$ is a result of the interference of probability waves, which is also the reason for the formation of multiple bright fringes. However, based on the orthogonality of the wave function [1], $\psi_{B} \psi_{A}{ }^{*}=0, \psi_{A} \psi_{B}{ }^{*}=0$; that is, the cross term does not exist at all, so the probabilistic Copenhagen interpretation cannot accurately explain the results of the double-slit experiment of photons or particles.

\section{New physical interpretation:}

Based on our new understanding of the wave function, in the double-slit experiment shown in Fig. 1(a), a photon or particle travels along direction $x$ after passing through slit A, and the position $x$ is determined by measurement. For a photon or particle in a QM system, the measurement of its position applies the position operator on the wave function of the photon or particle, i.e.,

$\hat{x} \psi=x_{A} \psi_{A}=x_{A} e^{i\left(p_{A} x_{A}-E_{A} t_{A}\right) / \hbar}$

Equation (16) shows that only when $p_{A} x_{A}=n h$ and $E_{A} t_{A}=n h$ can the measurement obtain a positive real value $x_{A}$. In other words, only when such space-time conditions are satisfied can the receiving screen detect photons or particles. For two photons or particles A and $\mathrm{B}$ with the same momentum and energy $\left(p_{A}=p_{B}=p, E_{A}=E_{B}=E\right)$ passing through the two slits (only one photon or particle for one slit) towards the receiving screen, which is used as the measurement instrument, only when the distance of A and B to the receiving screen follows Equations (17) and (18), respectively, can photons or particles be detected on the receiving screen. If two photons or particles reach the same point $\mathrm{P}$ on the receiving screen, then the path difference is $x_{A}-x_{B}=\left(n_{A}-n_{B}\right) h / p$. When the distance between the two slits $\mathrm{A}$ and $\mathrm{B}$ is $\mathrm{d}$, the distance between the two slits and the receiving screen is $\mathrm{L}$, and the distance between the point $\mathrm{P}$ on the receiving screen and the centre of the receiving screen is $y_{k}, x_{A}-x_{B}=d \sin \theta_{k}$ and $y_{k}=L \sin \theta_{k}$ according to the approximate geometric relationship of the photon or particle path. Considering that $n_{A}-n_{B}= \pm k, h / p=\lambda$, Equations (19) and (20) can be obtained.

$x_{A}=n_{A} h / p(17)$

$x_{B}=n_{B} h / p(18)$

$d \sin \theta_{k}= \pm k \lambda, \mathrm{k}=0,1,2,3 \ldots(19)$

$y_{k}=\frac{L}{d} \cdot( \pm k) \lambda, \mathrm{k}=0,1,2,3 \ldots$

Equations (19) and (20) are the new QM theoretical formulas for the double-slit experiments of photons and particles and are exactly the same as Equation (14) for the double-slit experiment of light. $\theta_{k}$ is the deviation angle of the position on the receiving screen. The bright spots of two photons or particles falling at the same position should simultaneously satisfy Equations (17), (18), (19), and (20).

Since the slit has a certain width, multiple photons or particles pass through the slit, and there should be a bright fringe with a certain width formed by the superposition of multiple bright spots on the receiving screen. When the photons or particles have different states, they have different wavelengths, and photons or particles of different states can be located in the different positions of the different bright fringes. The state of the photon or particle determines the position of the photon or particle in the same bright fringe, and the probability of a certain state of the particle determines only the position brightness of the particle in the bright fringe. Except 
for the bright fringes, the other areas are all dark fringes.

The double-slit experiment shows that whether the photons or particles arrive at the receiving screen one by one or arrive at the receiving screen together and whether the photons or particles come from double slits or a single slit or from any two different points, as long as Equations (17), (18), (19), and (20) are satisfied simultaneously, a photon or particle does not need to pass through two slits at the same time, and bright and dark fringes can appear. This is the result of a QM-based new physical interpretation and measurement theory about the particle state function, not the result of the probability.

During the motion of photons or particles, photons or particles periodically and alternately exhibit particle characteristics and wave characteristics. When the particle state occurs on the receiving screen, the receiving screen can detect the photon or particle, and the particle appears at the bright fringes on the receiving screen. When the wave state occurs on the receiving screen, the photons or particles cannot be detected on the receiving screen, the dark fringes on the receiving screen are the region of the wave state of the particles. In the state function of a photon or particle, the length of the spatial region that exhibits wave characteristics is the wavelength $\lambda$. Because this wavelength is very small, it is generally impossible to observe under macroscopic conditions. However, through the double-slit experiment, the spatial region that exhibits the wave characteristics of photons or particles on the receiving screen is magnified by $k \cdot L / d$ times and can be clearly observed under macroscopic conditions. Therefore, the result of the double-slit experiment is the geometric image of the wave-particle duality of the photon or particle being magnified by $k \cdot L / d$ times.

\subsection{Physical interpretation of the uncertainty principle \\ Copenhagen interpretation:}

In the QM system, when a single particle is measured, based on the state superposition principle of the wave function, the Born probability hypothesis, and the noncommutative relation of the operator, the Copenhagen interpretation obtains Equation (21) for the Heisenberg uncertainty relation after rigorous mathematical deduction. [11]

$\Delta x \cdot \Delta p \geq \hbar / 2(21)$

where $\Delta \mathrm{x}$ and $\Delta \mathrm{p}$ are the uncertainties of the position and momentum of a particle, respectively; the physical meaning of the uncertainty is that the position and momentum of a particle cannot be accurately determined at the same time, and there is no definite motion trajectory for microscopic particles. Although the uncertainty principle has become the basic principle of QM, its essential properties are still unclear.

However, from the mathematical derivation process of the uncertainty relation, we find that in Equation (21), $\Delta x=x_{j}-\bar{x}, \Delta p=p_{j}-\bar{p}, x_{j}$ and $p_{j}$ are all the one-time measurement of the position and momentum of a particle, i.e., the exact position and momentum of the particle obtained through the measurement, as shown in Equations (22) and (23); $\bar{x}$ and $\bar{p}$ are the averages of all possible positions and momentums of a particle in the QM system and can be calculated using Equations (24) and (25), respectively.

$\hat{x} \psi_{j}=x_{j} \psi_{j}(22)$

$\hat{p} \psi_{j}=p_{j} \psi_{j}(23)$

$\bar{x}=\iiint_{-\infty}^{+\infty} \psi^{*} \hat{x} \psi d x d y d z$

$\bar{p}=\iiint_{-\infty}^{+\infty} \psi^{*} \hat{p} \psi d x d y d z$ 
where $\hat{x}$ and $\hat{p}$ are the position operator and the momentum operator, respectively.

The above analysis shows that $\Delta x$ is the difference between the exact position and the average position of a particle and that $\Delta p$ is the difference between the exact momentum and the average momentum of a particle, that is, the standard deviation (SD). It is not appropriate to use the SD of the system to represent the measurement error of the position and momentum of a particle. More importantly, the position and momentum of the particle, $x_{j}$ and $p_{j}$, are obtained in this measurement, and the operator on the wave function is just the method of measurement; therefore, we cannot say that the measured value obtained this time is inaccurate, and it is inappropriate to state that the position and momentum of a particle cannot be accurately determined at the same time through Equation (21).

\section{New physical interpretation:}

(1) According to the derivation process of Equation (21) and the origin of the probability, if the position and momentum obtained from each measurement are different, it is inappropriate to state that the measurement is inaccurate, and the only sure thing is that the state and position of the particle have changed. In this way, Equation (21) can be rewritten as $\Delta x_{\left(x_{j} \rightarrow \bar{x}\right)}$. $\Delta p_{\left(p_{j} \rightarrow \bar{p}\right)} \geq \hbar / 2$, its physical meaning can be expressed as follows: the particle state of a particle is not continuous, and the product of the changes of the position and momentum of the particle when the particle transitions from the state $\mathrm{j}$ to the average state follows the rule of Equation (21); i.e., the minimum value of the product of the changes of the position and momentum is $\hbar / 2$.

(2) When the particle changes from the state $\mathrm{j}$ to the state $\mathrm{k}$, according to Planck-Einstein relation ${ }^{1}$, Equation $\Delta E_{j k}=E_{k}-E_{j}=h v_{j k}=h \frac{1}{\Delta t_{\mathrm{jk}}}$ is valid, that is, Equation (26) must be satisfied. Equation (26) shows that the action change of the particle must be equal to Planck's constant $\mathrm{h}$ when the particle changes from one particle state $\mathrm{j}$ to another particle state $\mathrm{k}$. Because the product of the changes of the position and momentum of the particle is also the action of the state change of the particle, Equation (27) must be satisfied.

$\Delta E_{j k} \cdot \Delta t_{j k}=h \quad(26)$

$\Delta p_{j k} \cdot \Delta x_{j k}=h \quad(27)$

where, $\Delta t_{\mathrm{jk}}$ and $\Delta x_{\mathrm{jk}}$ are the time and distance that the particle changes from the state $\mathrm{j}$ to the state $\mathrm{k}$, respectively, $\Delta E_{j k}$ and $\Delta p_{j k}$ are the changes of the energy and momentum that the particle changes from the state $\mathrm{j}$ to the state $\mathrm{k}$, respectively.

Equation (26) and (27) show that the particle state of a particle is not continuous, when the particle changes between any two particle states, the product of the changes of the position and momentum is $\mathrm{h}$, the product of the changes of the energy and the time is $\mathrm{h}$. Because the state function of the particle is $\psi_{j k}=C_{p_{j}} \psi_{j}+C_{p_{k}} \psi_{k}$ when the state of the particle changes between any two particle states, the particle is in the superposition (transition) state between two particle states, all mechanical quantities of the particle are uncertain.

The result of Equation (21) is not equal to $\mathrm{h}$ as Equation (27), the reason is that the particle in the average position and average momentum is generally not in a possible particle state. 


\section{New uncertainty relationship:}

We believe that in a quantum objective system, when a particle in state $\mathrm{j}$ is measured, the operator corresponding to the measured mechanical quantity is used to operate on the wave function of the particle. When the particle moves along the x-direction, its state function is Equation (28), and using any Hermitian operator $\hat{F}$ on Equation (28) can obtain Equation (29).

$\psi_{j}=e^{i\left(p_{j} \cdot x-E_{j} t\right) / \hbar}$

$\hat{F} \psi_{j}=F_{j} e^{i\left(p_{j} \cdot x-E_{j} t\right) / \hbar}$

where $F_{j}$ is the eigenvalue of the operator $\hat{F}$, which corresponds to the mechanical quantity of particle j, i.e., the real measured number of the mechanical quantity $F$.

Equation (29) shows that $\hat{F}$ is any Hermitian operator; that is, Equations (30) and (31), the conditions for measuring any mechanical quantity to obtain a positive real value, are valid.

$p_{j} x=n h, \mathrm{n}=1,2,3 \ldots(30)$

$E_{j} t=n h, \mathrm{n}=1,2,3 \ldots(31)$

In a QM system, Equations (30) and (31) can generally be expressed as Equation (32).

$\left\{\begin{array}{l}p x=n h \\ E t=n h\end{array}, \mathrm{n}=1,2,3 \ldots(32)\right.$

From the physical meaning of the particle state function, Equation (32) exactly gives the time-space points where a particle exhibits particle characteristics. Because the particle exhibits particle characteristics, all its mechanical quantities are determined and have determined values, including the momentum and position of the particle.

In the space-time region outside the space-time points of Equation (32), i.e., the space-time region represented by Equation (33), regardless of measurement or nonmeasurement, all the mechanical quantities of the particle are uncertain. According to the physical meaning of the particle state function, in this time-space region, the particle exhibits wave characteristics, and the wave does not have the same mechanical quantities as the particle, so Equation (33) becomes a new uncertainty relation.

$\left\{\begin{array}{l}(n-1) h<p x<n h \\ (n-1) h<E t<n h\end{array}, \mathrm{n}=1,2,3 \ldots\right.$

\subsection{Localization and nonlocality of a particle}

\subsubsection{Spatiotemporal ranges of particle localization and nonlocality}

The discontinuity of the particle characteristics of the particle can lead to the invalidity of the derivative of the mechanical quantity operator, which is also the essential reason for the noncommutative property of the two mechanical quantity operators. Although when a particle is in the particle state, all its mechanical quantities are determined, the measurement of the mechanical quantities related to the derivative, such as the momentum, energy, and angular momentum, is affected by the discontinuity of the particle nature; the measurement of these mechanical quantities must cross the time-space region of particle uncertainty, and its measurement accuracy is limited by the time-space region of particle discontinuity (wavelength and period) in Equation (33).

Based on the new uncertainty principle, we conclude that the particle is localized at the time-space points in Equation (32) in the particle state, and is not localized in the time-space region in Equation (33) in the wave state. Obviously, in a QM system, the nonlocalized 
time-space region of a particle is much larger than the localized time-space points, which may be the fundamental reason for the invalidity of Bell's inequality [12] in most cases.

\subsubsection{Apparent trajectory of a particle}

If the positions determined by Equation (32) are represented by dots, the dotted lines formed by these dots become the motion apparent trajectories of microscopic particles. At the point on the dotted line, the particle exhibits particle characteristics and can be observed. In the region between the two points, with a length of the wavelength $\lambda$, the particles exhibit wave characteristics, and no mechanical quantity is observed. When the geometric scale of the particle is greater than or equal to the spatial range of the volatility, $\lambda$, as shown in Equation (33), we can observe the particle only at the macrolevel, and the spatial region of volatility is covered. At this moment, the trajectory of the macroscopic particle looks like a solid line in Fig. 2(a) and (b).

\begin{tabular}{|c|c|}
\hline 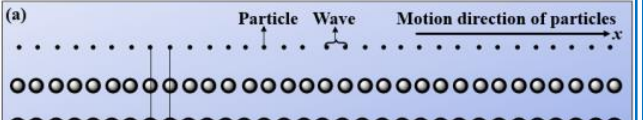 & 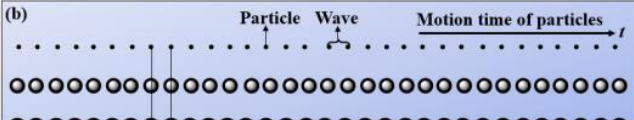 \\
\hline 000000000 & $\infty 00$ \\
\hline 00000000000000000000000000000000 & 000000000000000000000000000000 \\
\hline
\end{tabular}

Fig. 2 Motion apparent trajectories of particles

(a) Apparent trajectories in space;(b) apparent trajectories at time

\subsection{Physical interpretation of quantum tunnelling effect}

\section{Copenhagen interpretation:}

In a QM system, the wave function of a particle is obtained by solving the Schrödinger equation of a finite deep potential well. The Copenhagen interpretation indicates that the probability distribution density of a particle in space can be obtained through this wave function. The results show that when the energy of the particle is lower than the energy of the potential well, the particle has a certain probability to pass through the potential well, which is the quantum tunnelling effect $[13,14]$. Because the origin of the probability is not clear, it is difficult to understand the physical logic of particles with energies lower than the energy of the potential well passing through the well. Therefore, the reasonable explanation of the quantum tunnelling effect is not given by the Copenhagen interpretation.

\section{New physical interpretation:}

According to the origin of probability, we know that the energy of a particle in a QM system is the average energy of the particle calculated by Equation (34).

$\bar{E}=\iiint_{-\infty}^{+\infty} \psi^{*} \widehat{H} \psi d x d y d z(34)$

This average energy is the average energy of all particles in the quantum objective system. Obviously, of all the particles in a quantum objective system, some particles must have energies higher than the average energy $\bar{E}$. Of these particles with energies higher than the average, some particles can have energies higher than the potential energy $U$ of the potential well, and these particles can cross the energy barrier. In other words, for a particle in a QM system, when its energy eigenvalue is not only higher than the average energy but also higher than the potential energy $U$ of the potential well, the particle can cross the energy barrier, and the quantum tunnelling effect is the result of the energy eigenvalue of a particle being higher than the potential energy $U$ of the system.

\section{Conclusions}


(1) The superposition state of a particle state function can be viewed as the linear superposition of all allowed state functions in a multiparticle quantum objective system. Probability in QM originates from the particle number distribution rate in the unit volume near position $\mathrm{r}$ at time $\mathrm{t}$ in the multiparticle quantum objective system.

(2) The state function of a particle is the mathematical expression of its wave-particle duality, indicating that the particle periodically and alternately exhibits the particle state and wave state in time and space. The particle at time-space points of $p r=n h$ and $E t=n h(\mathrm{n}=1,2,3 \ldots)$ represents the particle state, and all the physical quantities of the particle are determined. In the time-space region of $(n-1) h<p r<n h,(\mathrm{n}-1) h<E t<n h(\mathrm{n}=1,2,3 \ldots)$, the particle exhibits the wave state, and all the mechanical quantities of the particle are uncertain.

(3) The double-slit experiment exhibits bright and dark fringes on the receiving screen, which is the geometric image of the wave-particle duality of the particle amplified by $k \cdot L / d$ times. The bright fringes are a collection of time-space points where multiple particles are in the particle state, and the dark fringes are the spatial region where particles are in the wave state.

(4) The new physical meaning of the Heisenberg uncertainty principle is that the particle state transition of a particle is discontinuous, when the particle state changes between any two particle states, the product of the changes of the position and momentum is always $h$, the product of the changes of the energy and the time is always $h$ too. The certainty relation of a particle is $p r=n h$ and $E t=n h(\mathrm{n}=1,2,3 \ldots)$, and at this time-space point, the particle is localized. The uncertainty relation of a particle is $(n-1) h<p r<n h,(\mathrm{n}-1) h<E t<n h$ $(\mathrm{n}=1,2,3 \ldots)$, and in this time-space region, the particle is nonlocalized.

\section{REFERENCES}

[1] Griffiths, David J. Introduction to Quantum Mechanics, Pearson Education Inc., 1995.

[2] Steven Weinbery, Why am I dissatisfied with quantum mechanics, Patrusky Lectre, Scientific American, Nov., 2016.

[3] A. Einstein, Über einen die erzeugung und verwandlung des lichtes betreffenden heuristischen gesichtspunkt. Ann. Phys.322, 132-148 (1905).

[4] A. Einstein, Concerning an Heuristic Point of View Towards the Emission and Transformation of Light, American Journal of Physics (Translation into English), v.33, n.5, May 1965.

[5] L. de Broglie, Waves and Quanta, Nature 112(1923) 540.

[6] M. Born, Quantum mechanics of collision processes, Zeit. Physik 38 (1926) 803.

[7] E. Schrödinger, Quantasierung als Eigenwertproblem, Ann. Der Physik 79(1926) 361.

[8] SJ Clark, Bohr's principle of complementarity, Ann Intern Med, 1988, Dec 15; 109(12): 994-5.

[9] Young T., Experiments and calculations relative to physical optics[C]. Phil Trans Roy Soc Lond, 1804, 94: $1-16$.

[10] A. Tonomura, J. Endo, T. Matsuda, and T Kawasaki, Demonstration of single-electron bulldup of an interference pattern, American Journal Phys., 57, 1989, 117.

[11] J. A. Wheeler and W. H. Zurek, Quantum Theory and Measurement, Princeton University Press, NJ, 1984, p.62.

[12] John S. Bell, On the Einstein Podolsky Rosen Paradox, Physics, 1, 1964, 195-200.

[13]Griffiths, David J. Introduction to Quantum Mechanics, Pearson Education Inc., 1995, p78-93.

[14] Razavy, Mohsen, Quantum Theory of Tunneling. World Scientific, 2003. 